\title{
La cirugía de revascularización miocárdica sumada al tratamiento médico reduce la mortalidad a diez años en pacientes con miocardiopatía isquémica
}

Coronary-Artery Bypass Surgery added to medical therapy reduces the ten year mortality of patients with ischemic cardiomyopathy

\section{Objetivos}

Comparar si la adición de la cirugía de revascularización miocárdica (CRM) al tratamiento médico en la miocardiopatía isquémica mejora los resultados a los diez años de seguimiento.

\section{Diseño, lugar y participantes}

Prolongación del seguimiento prolongado del estudio STICH, ensayo clínico aleatorizado abierto multicéntrico (127 centros en 26 países). ${ }^{1}$

Se incluyeron a pacientes con insuficiencia cardiaca y fracción de eyección reducida $(<35 \%)$, enfermedad coronaria de múltiples vasos (angina en $\mathrm{CF}>2$ ), pasibles de ser revascularizados. Se excluyeron los pacientes con enfermedad de tronco de la coronaria izquierda $>50 \%$ y angina de reposo bajo tratamiento médico completo, enfermedad valvular aórtica con indicación de cirugía y shock cardiogénico dentro de las 72 horas.

Tabla 1. Resultados primarios y secundarios
Velazquez EJ, y col. N Engl J Med 2016;374:1511-20.

\section{Intervención}

Los pacientes fueron aleatorizados a CRM en adición al tratamiento médico óptimo o tratamiento médico óptimo aislado.

\section{Medición de los resultados principales}

El resultado primario fue muerte por cualquier causa y los secundarios fueron muerte cardiovascular; y el combinado de muerte y re-internaciones de causa cardiaca. Los resultados fueron analizados según intención de tratar.

\section{Resultados Principales}

Tras una mediana de seguimiento de 9,8 años, la mortalidad del grupo CRM fue menor que en el grupo de tratamiento médico aislado (Tabla 1). Los resultados para el punto final primario, se mantuvieron en el análisis por protocolo (este análisis contemplo a los 536 pacientes del grupo tratamiento médico que no se cruzaron al tratamiento quirúrgico dentro del año y a los 555 pacientes del grupo quirúrgico operados dentro de ese mismo periodo)

\begin{tabular}{|c|c|c|c|c|}
\hline Resultado & $\begin{array}{c}\text { CRM } \\
n=610(\%)\end{array}$ & $\begin{array}{c}\text { Tratamiento médico } \\
n=602(\%)\end{array}$ & $\begin{array}{l}\text { Hazard Ratio } \\
(\mathrm{IC9} 5 \%)\end{array}$ & $\mathbf{P}$ \\
\hline \multicolumn{5}{|l|}{ Primario } \\
\hline Muerte por cualquier causa & $359(58,9 \%)$ & $398(66,1 \%)$ & $0,84(0,73$ a 0,97$)$ & 0,02 \\
\hline \multicolumn{5}{|l|}{ Secundarios } \\
\hline Muerte Cardiovascular & $247(40,5 \%)$ & $297(49,3 \%)$ & $0,79(0,66$ a 0,93$)$ & 0,006 \\
\hline Muerte y re-internaciones de causa cardiaca & $467(76,6 \%)$ & $524(49,3 \%)$ & $0,72(0,64$ a 0,82$)$ & $<0,001$ \\
\hline
\end{tabular}

CRM: cirugía de revascularización miocárdica. IC 95\%: intervalo de confianza del 95\%.

Conclusiones

En una cohorte de pacientes con miocardiopatía isquémica se- guidos por 10 años, la CRM, en adición al tratamiento médico óptimo, redujo la muerte total y cardiovascular.

\section{Comentario}

Este estudio agrega la CRM a la lista de estrategias farmacológicas (beta bloqueantes, inhibidores de la enzima convertidora de angiotensina, anti-aldosterónicos, antagonistas de los receptores de angiotensina II/Sacubitril) y no farmacológicas (dispositivos) que han logrado beneficio clínico en términos de reducción de la mortalidad total de esta enfermedad epidémica. ${ }^{2,3}$ En términos absolutos habría que realizar $14 \mathrm{CRM}$, más allá del tratamiento médico óptimo, para evitar una muerte en 10 años.

Este efecto es extrapolable, en tanto y en cuanto nuestro paciente en cuestión sea similar a la población de este estudio (pacientes jóvenes, sin insuficiencia renal, con disminución de la fracción de eyección y no operados previamente), y que además se repliquen los resultados quirúrgicos que reportan los autores, es decir, una mortalidad observada del 3,6\% (menor a la esperada según la definición del protocolo).

\section{Conclusiones del comentador}

Al escepticismo del estudio primitivo a cinco años de seguimiento con resultado neutro, le siguen estos resultados favorables a la CRM que las nuevas guías deberán considerar en sus actualizaciones.

\section{Aníbal Arias [ Servicio de Cardiología del Hospital Italiano de Buenos Aires anibal.arias@ hospitalitaliano.org.ar ]}

Arias A. La cirugía de revascularización miocárdica sumada al tratamiento médico reduce la mortalidad a diez años en pacientes con miocardiopatía isquémica.Evid Act Pract Ambul. 2017;20(2):40. Comentado de: Velazquez EJ, y col. Coronary-Artery Bypass Surgery in Patients with Ischemic Cardiomyopathy. N Engl J Med. 2016;374(16):1511-20. PMID: 27040723.

\section{Referencias}

1. Velazquez EJ y col. Coronary-artery bypass surgery in patients with left ventricular dysfunction. N Engl J Med 2011:364:1607-16.

2. Fihn SD y col. 2012 ACCF/AHA/ACP/ AATS/PCNA/SCAI/STS Guideline for the diagnosis and management of patients with stable ischemic heart disease: a report of the American College of Cardiology Foundation/American Heart Association Task Force on Practice Guidelines, and the American College of Physicians, American Association for Thoracic Surgery, Preventive Cardiovascular Nurses Association, Society for Cardiovascular Angiography and Interventions, and Society of Thoracic Surgeons. J Am Coll Cardiol 2012;60(24):e44-e164

3. McMurray JJ, y col. Angiotensin-Neprilysin Inhibition versus Enalapril in Heart Failure. N Engl J Med 2014;371:993-1004. 